\title{
Dynamics of a stochastic non-autonomous predator-prey system with Beddington-DeAngelis functional response
}

Shuang $\mathrm{Li}^{1,2^{*}}$ and Xinan Zhang ${ }^{1}$

\section{"Correspondence:}

oklishuang@yahoo.com.cn

'School of Mathematics and

Statistics, Central China Normal

University, Wuhan, Hubei 430079,

P.R. China

${ }^{2}$ Department of Mathematics, Xinxiang University, Xinxiang,

Henan 453000, P.R. China

\begin{abstract}
A stochastic non-autonomous predator-prey system with Beddington-DeAngelis functional response is proposed, the existence of a global positive solution and stochastically ultimate boundedness are derived. Sufficient conditions for extinction, non-persistence in the mean, weak persistence in the mean and strong persistence in the mean are established. The global attractiveness of the solution is also considered. Finally, numerical simulations are carried out to support our findings.
\end{abstract}

MSC: 92B05; 34F05; 60H10; 93E03

Keywords: predator-prey; non-autonomous; stochastic; Beddington-DeAngelis; persistence; extinction

\section{Introduction}

In population dynamics, the relationship between predator and prey plays an important role due to its universal existence. There are many significant functional responses in order to model various different situations. In fact, most of the functional responses are prey-dependent; however, the functional response should also be predator-dependent, especially when predators have to search for food. Beddington [1] and DeAngelis et al. [2] in 1975 first introduced the Beddington-DeAngelis type predator-prey model taking the form

$$
\left\{\begin{array}{l}
\frac{d u}{d t}=r_{1} u-g_{1} u^{2}-\frac{f_{1} u v}{\beta \beta \gamma u+\delta v}, \\
\frac{d v}{d t}=r_{2} v-g_{2} v^{2}+\frac{f_{2} u v}{\beta+\gamma u+\delta v}
\end{array}\right.
$$

where $u$ and $v$ denote the population densities of prey and predator. Although the Beddington-DeAngelis functional response is similar to the Holling type-II functional, it can reflect mutual interference among predators. That is to say, this kind of functional response is affected by both predator and prey. In the last years, some experts have studied the system [3-7]. In the following, we introduce a non-autonomous predator-prey model with Beddington-DeAngelis functional response:

$$
\left\{\begin{array}{l}
\frac{d x}{d t}=x\left(a_{1}(t)-b_{1}(t) x-\frac{c_{1}(t) y}{m_{1}(t)+m_{2}(t) x+m_{3}(t) y}\right), \\
\frac{d y}{d t}=y\left(-a_{2}(t)-b_{2}(t) y+\frac{c_{2}(t) x}{m_{1}(t)+m_{2}(t) x+m_{3}(t) y}\right),
\end{array}\right.
$$

(c) $2013 \mathrm{Li}$ and Zhang; licensee Springer. This is an Open Access article distributed under the terms of the Creative Commons Attribution License (http://creativecommons.org/licenses/by/2.0), which permits unrestricted use, distribution, and reproduction in any medium, provided the original work is properly cited. 
where $x(t)$ and $y(t)$ represent the population density of prey and predator at time $t$, respectively. $a_{1}(t)$ denotes the intrinsic growth rate of prey. $b_{1}(t)$ and $b_{2}(t)$ stand for the density-dependent coefficients of prey and predator, respectively. $a_{2}(t)$ is the death rate of predator. $c_{1}(t)$ is the capturing rate of predator, $c_{2}(t)$ represents the rate of conversion of nutrients into the reproduction of predator. For the other coefficients' biological representation, we refer the reader to [1] and [2].

On the other hand, population systems are often subject to environmental noise; therefore, it is important to study how the noise affects the population systems. In fact, stochastic population systems have been studied recently by many authors [8-17]. However, there are not many papers considering non-autonomous stochastic systems [18-23]. In this paper, considering the effect of environmental noise, we introduce stochastic perturbation into the intrinsic growth rate of prey and the death rate of predator in system (2) and assume the parameters $a_{1}(t)$ and $a_{2}(t)$ are disturbed to $a_{1}(t)+\alpha(t) d B_{1}(t),-a_{2}(t)+\beta(t) d B_{2}(t)$, respectively. Then corresponding to the deterministic system (2), we obtain the following stochastic system:

$$
\left\{\begin{array}{l}
d x=x\left(a_{1}(t)-b_{1}(t) x-\frac{c_{1}(t) y}{m_{1}(t)+m_{2}(t) x+m_{3}(t) y}\right) d t+\alpha(t) x d B_{1}(t), \\
d y=y\left(-a_{2}(t)-b_{2}(t) y+\frac{c_{2}(t) x}{m_{1}(t)+m_{2}(t) x+m_{3}(t) y}\right) d t+\beta(t) y d B_{2}(t) .
\end{array}\right.
$$

We assume all the coefficients are continuous bounded nonnegative functions on $R_{+}=$ $[0,+\infty)$.

If $f(t)$ is a continuous bounded function on $R_{+}$, define

$$
f^{u}=\sup _{t \in R_{+}} f(t), \quad f^{l}=\inf _{t \in R_{+}} f(t) .
$$

Throughout this paper, suppose that $a_{2}^{l}>0, m_{i}^{l}>0(i=1,2,3), b_{i}^{l}>0(i=1,2)$.

Definition 1 (1) The population $x(t)$ is said to be non-persistent in the mean if $\langle x\rangle^{*}=0$, where $\langle f(t)\rangle=\frac{1}{t} \int_{0}^{t} f(s) d s, f^{*}=\lim \sup _{t \rightarrow+\infty} f(t), f_{*}=\liminf _{t \rightarrow+\infty} f(t)$.

(2) The population $x(t)$ is said to be weakly persistent in the mean if $\langle x\rangle^{*}>0$.

(3) The population $x(t)$ is said to be strongly persistent in the mean if $\langle x\rangle_{*}>0$.

Throughout this paper, unless otherwise specified, let $(\Omega, \mathcal{F}, \mathcal{P})$ be a complete probability space with a filtration $\left\{\mathcal{F}_{t}\right\}_{t \in R}$ satisfying the usual conditions (i.e., it is right continuous and increasing and $\mathcal{F}_{0}$ contains all $\mathcal{P}$-null sets), here $\dot{B}_{i}(t)(i=1,2)$ is a standard Brownian motion defined on this probability space. In addition, $R_{+}^{2}$ denotes $\left\{(x, y) \in R^{2}: x>0, y>0\right\}$.

Here we give the following auxiliary statements which are introduced in [24]. Consider the $d$-dimensional stochastic differential equation

$$
d x(t)=f(x(t), t) d t+g(x(t), t) d B(t), \quad t \geq t_{0} .
$$

Denote by $C^{2,1}\left(R^{d} \times\left[t_{0}, \infty\right) ; R_{+}\right)$the family of all nonnegative functions $V(x, t)$ defined on $R^{d} \times\left[t_{0}, \infty\right)$ such that they are continuously twice differentiable in $x$ and once in $t$. The differential operator $L$ of the above equation is defined by the formula

$$
L=\frac{\partial}{\partial t}+\sum_{i=1}^{d} f_{i}(x, t) \frac{\partial}{\partial x_{i}}+\frac{1}{2} \sum_{i, j=1}^{d}\left[g^{T}(x, t) g(x, t)\right]_{i j} \frac{\partial^{2}}{\partial x_{i} \partial x_{i}} .
$$


If $L$ acts on a function $V \in C^{2,1}\left(R^{d} \times\left[t_{0}, \infty\right) ; R_{+}\right)$, then

$$
L V(x, t)=V_{t}(x, t)+V_{x}(x, t) f(x, t)+\frac{1}{2} \operatorname{trace}\left[g^{T}(x, t) V_{x x}(x, t) g(x, t)\right]
$$

\section{Global positive solution and stochastic boundedness}

For a model of population dynamics, the first thing considered is whether the solution is globally existent and nonnegative, hence in this section we will show it. In order for a stochastic differential equation to have a unique global solution for any given initial value, the coefficients of the equation are generally required to satisfy the linear growth condition and the local Lipschitz condition [24]. The coefficients of model (3) do not satisfy the linear growth condition, though they are locally Lipschitz continuous, the solution of system (3) may explode at a finite time ( $c f$. [24]). In the following, using the Lyapunov analysis method, we can prove that system (3) has a global positive solution.

Theorem 1 For any initial value $\left(x_{0}, y_{0}\right) \in R_{+}^{2}$, there is a unique solution $(x(t), y(t))$ of system (3) on $t \geq 0$, and the solution will remain in $R_{+}^{2}$ with probability 1.

Proof Since the coefficients of model (3) are locally Lipschitz continuous, for any given initial value $\left(x_{0}, y_{0}\right) \in R_{+}^{2}$, there is a unique local solution $(x(t), y(t))$ on $t \in\left[0, \tau_{e}\right)$, where $\tau_{e}$ is the explosion time [24]. To show the solution is global, we need to show that $\tau_{e}=\infty$.

Let $n_{0}>0$ be sufficiently large for $x_{0}$ and $y_{0}$ lying within the interval $\left[1 / n_{0}, n_{0}\right]$. For each integer $n>n_{0}$, we define the stopping times

$$
\tau_{n}=\inf \left\{t \in\left[0, \tau_{e}\right): x(t) \notin(1 / n, n) \text { or } y(t) \notin(1 / n, n)\right\}
$$

where, throughout this paper, we set $\inf \emptyset=\infty$ ( $\varnothing$ denotes the empty set). Obviously, $\tau_{n}$ is increasing as $n \rightarrow \infty$. Let $\tau_{\infty}=\lim _{n \rightarrow \infty} \tau_{n}$, hence, $\tau_{\infty} \leq \tau_{e}$ a.s. Now, we only need to show $\tau_{\infty}=\infty$. If this statement is false, there is a pair of constants $T>0$ and $\varepsilon \in(0,1)$ such that $P\left\{\tau_{\infty} \leq T\right\}>\varepsilon$. Consequently, there exists an integer $n_{1} \geq n_{0}$ such that

$$
P\left\{\tau_{n} \leq T\right\}>\varepsilon, \quad n \geq n_{1}
$$

Define a $C^{2}$-function $V: R_{+}^{2} \rightarrow R_{+}$by

$$
V(x, y)=(x-1-\log x)+(y-1-\log y) .
$$

The nonnegativity of this function can be seen from $u-1-\log u \geq 0, \forall u>0$. Applying Itô's formula, we get

$$
\begin{aligned}
d V= & (x-1)\left(a_{1}(t)-b_{1}(t) x-\frac{c_{1}(t) y}{m_{1}(t)+m_{2}(t) x+m_{3}(t) y}\right) d t+\frac{\alpha^{2}(t)}{2} d t \\
& +(y-1)\left(-a_{2}(t)-b_{2}(t) y+\frac{c_{2}(t) x}{m_{1}(t)+m_{2}(t) x+m_{3}(t) y}\right) d t+\frac{\beta^{2}(t)}{2} d t \\
& +(x-1) \alpha(t) d B_{1}(t)+(y-1) \beta(t) d B_{2}(t),
\end{aligned}
$$


then

$$
\begin{aligned}
L V= & a_{1}(t) x-b_{1}(t) x^{2}-\frac{c_{1}(t) x y}{m_{1}(t)+m_{2}(t) x+m_{3}(t) y} \\
& -a_{1}(t)+b_{1}(t) x+\frac{c_{1}(t) y}{m_{1}(t)+m_{2}(t) x+m_{3}(t) y} \\
& +\frac{\alpha^{2}(t)}{2}-a_{2}(t) y-b_{2}(t) y^{2}+\frac{c_{2}(t) x y}{m_{1}(t)+m_{2}(t) x+m_{3}(t) y}+a_{2}(t)+b_{2}(t) y \\
& -\frac{c_{2}(t) x}{m_{1}(t)+m_{2}(t) x+m_{3}(t) y}+\frac{\beta^{2}(t)}{2} \\
\leq & a_{1}(t) x-b_{1}(t) x^{2}-a_{1}(t)+b_{1}(t) x+\frac{c_{1}(t) y}{m_{1}(t)+m_{2}(t) x+m_{3}(t) y}+\frac{\alpha^{2}(t)}{2} \\
& -a_{2}(t) y-b_{2}(t) y^{2}+\frac{c_{2}(t) x y}{m_{1}(t)+m_{2}(t) x+m_{3}(t) y}+a_{2}(t)+b_{2}(t) y+\frac{\beta^{2}(t)}{2} \\
\leq & a_{1}^{u} x-b_{1}^{l} x^{2}-a_{1}^{l}+b_{1}^{u} x+\frac{c_{1}^{u}}{m_{3}^{l}}+\frac{\left(\alpha^{u}\right)^{2}}{2}-a_{2}^{l} y-b_{2}^{l} y^{2}+\frac{c_{2}^{u}}{m_{2}^{l}} y+a_{2}^{u}+b_{2}^{u} y+\frac{\left(\beta^{u}\right)^{2}}{2} \\
\leq & \left(a_{1}^{u}+b_{1}^{u}\right) x-b_{1}^{l} x^{2}-a_{1}^{l}+\frac{c_{1}^{u}}{m_{3}^{l}}+\frac{\left(\alpha^{u}\right)^{2}}{2} \\
& +\left(b_{2}^{u}+\frac{c_{2}^{u}}{m_{2}^{l}}-a_{2}^{l}\right) y-b_{2}^{l} y^{2}+a_{2}^{u}+\frac{\left(\beta^{u}\right)^{2}}{2} \\
\leq & K,
\end{aligned}
$$

where $K$ is a positive number. Substituting this inequality into Eq. (5), we see that

$$
d V(x(t), y(t)) \leq K d t+(x-1) \alpha(t) d B_{1}(t)+(y-1) \beta(t) d B_{2}(t)
$$

which implies that

$$
\begin{aligned}
\int_{0}^{\tau_{n} \wedge T} d V(x(t), y(t)) \leq & \int_{0}^{\tau_{n} \wedge T} K d t+\int_{0}^{\tau_{n} \wedge T} \alpha(s)(x(s)-1) d B_{1}(s) \\
& +\int_{0}^{\tau_{n} \wedge T} \beta(s)(y(s)-1) d B_{2}(s),
\end{aligned}
$$

where $\tau_{n} \wedge T=\min \left\{\tau_{n}, T\right\}$. Taking the expectations of the above inequality leads to

$$
E V\left(x\left(\tau_{n} \wedge T\right), y\left(\tau_{n} \wedge T\right)\right) \leq V\left(x_{0}, y_{0}\right)+K E\left(\tau_{n} \wedge T\right) \leq V\left(x_{0}, y_{0}\right)+K T
$$

Let $\Omega_{n}=\left\{\tau_{n} \leq T\right\}$ for $n \geq n_{1}$, then by (4) we have $P\left(\Omega_{n}\right) \geq \varepsilon$. Note that for every $\omega \in \Omega_{n}$, there is at least one of $x\left(\tau_{n}, \omega\right)$ and $y\left(\tau_{n}, \omega\right)$ equaling either $n$ or $\frac{1}{n}$; therefore, $V\left(x\left(\tau_{n}, \omega\right), y\left(\tau_{n}, \omega\right)\right)$ is no less than $\min \left\{(n-1-\log n),\left(\frac{1}{n}-1-\log \frac{1}{n}\right)\right\}$. It then follows from (6) that

$$
\begin{aligned}
V\left(x_{0}, y_{0}\right)+K T & \geq E\left[1_{\Omega_{n}(\omega)} V\left(x\left(\tau_{n}\right), y\left(\tau_{n}\right)\right)\right] \\
& \geq \varepsilon \min \left\{(n-1-\log n),\left(\frac{1}{n}-1-\log \frac{1}{n}\right)\right\},
\end{aligned}
$$


where $1_{\Omega_{n}(\omega)}$ is the indicator function of $\Omega_{n}$, letting $n \rightarrow \infty$, we have that

$$
\infty>V\left(x_{0}, y_{0}\right)+K T=\infty
$$

is a contradiction, then we must have $\tau_{\infty}=\infty$. Therefore, the solution of system (3) will not explode at a finite time with probability 1 . This completes the proof.

Definition 2 The solution $X(t)=(x(t), y(t))$ of system (3) is said to be stochastically ultimately bounded if for any $\varepsilon \in(0,1)$, there is a positive constant $\chi(\chi(\varepsilon))$ such that for any initial value $\left(x_{0}, y_{0}\right) \in R_{+}^{2}$, the solution of (3) has the property that

$$
\limsup _{t \rightarrow \infty} P\{|X(t)|>\chi\}<\varepsilon
$$

Lemma 1 Let $(x(t), y(t))$ be a solution of system (3) with initial value $\left(x_{0}, y_{0}\right) \in R_{+}^{2}$, for all $p>1$, there exist $K_{1}(p)$ and $K_{2}(p)$ such that

$$
E\left[x^{p}(t)\right] \leq K_{1}(p), \quad E\left[y^{p}(t)\right] \leq K_{2}(p), \quad t \in[0,+\infty),
$$

where $K_{1}(p)=\max \left\{\bar{K}_{1}(p),\left(\frac{a_{1}^{u}+\frac{1}{2} p\left(\alpha^{u}\right)^{2}}{b_{1}^{l}}\right)^{p}+\tilde{\varepsilon}\right\}, K_{2}(p)=\max \left\{\bar{K}_{2}(p),\left(\frac{\frac{c_{2}^{u}}{m_{2}^{l}}+\frac{1}{2} p\left(\beta^{u}\right)^{2}}{b_{2}^{l}}\right)^{p}+\tilde{\varepsilon}\right\}, \bar{K}_{i}(p)(i=$ $1,2)$ and $\tilde{\varepsilon}$ are both positive constants.

Proof Define $V_{1}(x)=x^{p}$ for $x \in R_{+}$, where $p>1$. Applying Itô's formula leads to

$$
\begin{aligned}
d V_{1}(x)= & p x^{p-1} d x+\frac{1}{2} p(p-1) x^{p-2}(d x)^{2} \\
= & p x^{p}\left[a_{1}(t)-b_{1}(t) x-\frac{c_{1}(t) y}{m_{1}(t)+m_{2}(t) x+m_{3}(t) y}+\frac{1}{2}(p-1) \alpha^{2}(t)\right] d t \\
& +p x^{p} \alpha(t) d B_{1}(t) \\
\leq & p x^{p}\left[a_{1}^{u}+\frac{1}{2} p\left(\alpha^{u}\right)^{2}-b_{1}^{l} x\right] d t+p x^{p} \alpha(t) d B_{1}(t) .
\end{aligned}
$$

Similarly, we have

$$
\begin{aligned}
d y^{p}= & p y^{p}\left[-a_{2}(t)-b_{2}(t) y+\frac{c_{2}(t) x}{m_{1}(t)+m_{2}(t) x+m_{3}(t) y}+\frac{1}{2}(p-1) \beta^{2}(t)\right] d t \\
& +p y^{p} \beta(t) d B_{2}(t) \\
\leq & p y^{p}\left[\frac{c_{2}^{u}}{m_{2}^{l}}+\frac{1}{2} p\left(\beta^{u}\right)^{2}-b_{2}^{l} y\right] d t+p y^{p} \beta(t) d B_{2}(t) .
\end{aligned}
$$

Taking expectation, we have

$$
\begin{aligned}
\frac{d E\left[x^{p}(t)\right]}{d t} & \leq p\left\{\left[a_{1}^{u}+\frac{1}{2} p\left(\alpha^{u}\right)^{2}\right] E\left[x^{p}(t)\right]-b_{1}^{l} E\left[x^{p+1}(t)\right]\right\} \\
& \leq p\left\{\left[a_{1}^{u}+\frac{1}{2} p\left(\alpha^{u}\right)^{2}\right] E\left[x^{p}(t)\right]-b_{1}^{l}\left[E\left[x^{p}(t)\right]\right]^{1+\frac{1}{p}}\right\} \\
& \leq E\left[x^{p}(t)\right] p\left\{\left[a_{1}^{u}+\frac{1}{2} p\left(\alpha^{u}\right)^{2}\right]-b_{1}^{l}\left[E\left[x^{p}(t)\right]\right]^{\frac{1}{p}}\right\}
\end{aligned}
$$


and

$$
\begin{aligned}
\frac{d E\left[y^{p}(t)\right]}{d t} & \leq p\left\{\left[\frac{c_{2}^{u}}{m_{2}^{l}}+\frac{1}{2} p\left(\beta^{u}\right)^{2}\right] E\left[y^{p}(t)\right]-b_{2}^{l} E\left[y^{p+1}(t)\right]\right\} \\
& \leq p\left\{\left[\frac{c_{2}^{u}}{m_{2}^{l}}+\frac{1}{2} p\left(\beta^{u}\right)^{2}\right] E\left[y^{p}(t)\right]-b_{2}^{l}\left[E\left[y^{p}(t)\right]\right]^{1+\frac{1}{p}}\right\} \\
& \leq E\left[y^{p}(t)\right] p\left\{\left[\frac{c_{2}^{u}}{m_{2}^{l}}+\frac{1}{2} p\left(\beta^{u}\right)^{2}\right]-b_{2}^{l}\left[E\left[y^{p}(t)\right]\right]^{\frac{1}{p}}\right\} .
\end{aligned}
$$

Therefore, by the comparison theorem, we get

$$
\limsup _{t \rightarrow \infty} E\left[x^{p}(t)\right] \leq\left(\frac{a_{1}^{u}+\frac{1}{2} p\left(\alpha^{u}\right)^{2}}{b_{1}^{l}}\right)^{p}, \quad \limsup _{t \rightarrow \infty} E\left[y^{p}(t)\right] \leq\left(\frac{\frac{c_{2}^{u}}{m_{2}^{l}}+\frac{1}{2} p\left(\beta^{u}\right)^{2}}{b_{2}^{l}}\right)^{p} .
$$

Thus, for a given constant $\tilde{\varepsilon}>0$, there exists a $T>0$ such that for all $t>T$,

$$
E\left[x^{p}(t)\right] \leq\left(\frac{a_{1}^{u}+\frac{1}{2} p\left(\alpha^{u}\right)^{2}}{b_{1}^{l}}\right)^{p}+\tilde{\varepsilon}, \quad E\left[y^{p}(t)\right] \leq\left(\frac{\frac{c_{2}^{u}}{m_{2}^{l}}+\frac{1}{2} p\left(\beta^{u}\right)^{2}}{b_{2}^{l}}\right)^{p}+\tilde{\varepsilon} .
$$

Together with the continuity of $E\left[x^{p}(t)\right]$ and $E\left[y^{p}(t)\right]$, there exist $\bar{K}_{1}(p)>0, \bar{K}_{2}(p)>0$ such that $E\left[x^{p}(t)\right] \leq \bar{K}_{1}(p), E\left[y^{p}(t)\right] \leq \bar{K}_{2}(p)$ for $t \leq T$. Let

$$
\begin{aligned}
& K_{1}(p)=\max \left\{\bar{K}_{1}(p),\left(\frac{a_{1}^{u}+\frac{1}{2} p\left(\alpha^{u}\right)^{2}}{b_{1}^{l}}\right)^{p}+\tilde{\varepsilon}\right\}, \\
& K_{2}(p)=\max \left\{\bar{K}_{2}(p),\left(\frac{\frac{c_{2}^{u}}{m_{2}^{l}}+\frac{1}{2} p\left(\beta^{u}\right)^{2}}{b_{2}^{l}}\right)^{p}+\tilde{\varepsilon}\right\},
\end{aligned}
$$

then for all $t \in R_{+}$,

$$
E\left[x^{p}(t)\right] \leq K_{1}(p), \quad E\left[y^{p}(t)\right] \leq K_{2}(p) .
$$

Theorem 2 The solutions of system (3) with initial value $\left(x_{0}, y_{0}\right) \in R_{+}^{2}$ are stochastically ultimately bounded.

Proof If $X=(x, y) \in R^{2}$, its norm here is denoted by $|X|=\left(x^{2}+y^{2}\right)^{\frac{1}{2}}$, then

$$
|X(t)|^{p} \leq 2^{\frac{p}{2}}\left(|x(t)|^{p}+|y(t)|^{p}\right)
$$

by Lemma $1, E|X(t)|^{p} \leq K(p), t \in(0,+\infty) . K(p)$ is dependent of $\left(x_{0}, y_{0}\right)$ and defined by $K(p)=2^{\frac{p}{2}}\left(K_{1}(p)+K_{2}(p)\right)$. By virtue of Chebyshev's inequality, the above result is straightforward.

\section{Persistence in the mean and extinction}

Lemma 2 The solutions of system (3) with initial value $\left(x_{0}, y_{0}\right) \in R_{+}^{2}$ have the following properties:

$$
\limsup _{t \rightarrow \infty} \frac{\ln x(t)}{t} \leq 0, \quad \limsup _{t \rightarrow \infty} \frac{\ln y(t)}{t} \leq 0, \quad \text { a.s. }
$$


Proof It follows from system (3) that

$$
\left\{\begin{array}{l}
d x \leq x\left(a_{1}(t)-b_{1}(t) x\right) d t+\alpha(t) x d B_{1}(t), \\
d y \leq y\left(\frac{c_{2}(t)}{m_{2}(t)}-b_{2}(t) y\right) d t+\beta(t) y d B_{2}(t) .
\end{array}\right.
$$

Set

$$
\left\{\begin{array}{l}
d \bar{x}=\bar{x}\left(a_{1}(t)-b_{1}(t) \bar{x}\right) d t+\alpha(t) \bar{x} d B_{1}(t), \\
d \bar{y}=\bar{y}\left(\frac{c_{2}(t)}{m_{2}(t)}-b_{2}(t) \bar{y}\right) d t+\beta(t) \bar{y} d B_{2}(t),
\end{array}\right.
$$

where $(\bar{x}(t), \bar{y}(t))$ is a solution of system (8) with initial value $x_{0}>0$ and $y_{0}>0$. By the comparison theorem for stochastic differential equations, it is easy to have

$$
x(t) \leq \bar{x}(t), \quad y(t) \leq \bar{y}(t), \quad \text { a.s. } t \in[0,+\infty) .
$$

By Lemma 3.4 in [20], it is easy to get the following result:

$$
d x(t)=x(t)\left[\left(b(t)-a_{11}(t) x(t)\right) d t+\sigma(t) d B(t)\right]
$$

here $b(t), a_{11}(t)$ and $\sigma(t)$ are all nonnegative functions defined on $R_{+}$. If $a_{11}^{l}>0$, then $\lim \sup _{t \rightarrow \infty} \frac{\ln (|x(t)|)}{\ln t} \leq 1$, a.s.

Note that $b_{i}^{l}>0(i=1,2)$, then it follows from Eqs. (8) and (9),

$$
\limsup _{t \rightarrow \infty} \frac{\ln x(t)}{\ln t} \leq \limsup _{t \rightarrow \infty} \frac{\ln \bar{x}(t)}{\ln t} \leq 1, \quad \limsup _{t \rightarrow \infty} \frac{\ln y(t)}{\ln t} \leq \limsup _{t \rightarrow \infty} \frac{\ln \bar{y}(t)}{\ln t} \leq 1, \quad \text { a.s. }
$$

In addition,

$$
\limsup _{t \rightarrow \infty} \frac{\ln x(t)}{t}=\limsup _{t \rightarrow \infty} \frac{\ln x(t)}{\ln t} \limsup _{t \rightarrow \infty} \frac{\ln t}{t} \leq \limsup _{t \rightarrow \infty} \frac{\ln t}{t}=0
$$

therefore, it leads to $\lim \sup _{t \rightarrow \infty} \frac{\ln x(t)}{t} \leq 0$, a.s. Similarly, we can have $\lim \sup _{t \rightarrow \infty} \frac{\ln y(t)}{t} \leq 0$, a.s.

Lemma 3 [25] Suppose that $x(t) \in C\left[\Omega \times R_{+}, R_{+}^{0}\right]$, where $R_{+}^{0}:=\{a \mid a>0, a \in R\}$.

(I) If there are positive constants $\lambda_{0}, T$ and $\lambda \geq 0$ such that

$$
\ln x(t) \leq \lambda t-\lambda_{0} \int_{0}^{t} x(s) d s+\sum_{i=1}^{n} \beta_{i} B_{i}(t)
$$

for $t \geq T$, where $\beta_{i}$ is a constant $1 \leq i \leq n$, then $\langle x\rangle^{*} \leq \lambda / \lambda_{0}$, a.s. (i.e., almost surely).

(II) If there are positive constants $\lambda_{0}, T$ and $\lambda \geq 0$ such that

$$
\ln x(t) \geq \lambda t-\lambda_{0} \int_{0}^{t} x(s) d s+\sum_{i=1}^{n} \beta_{i} B_{i}(t)
$$

for $t \geq T$, where $\beta_{i}$ is a constant $1 \leq i \leq n$, then $\langle x\rangle_{*} \geq \lambda / \lambda_{0}$, a.s. 
In the following, we give the result about weak persistence in the mean and extinction of the prey and predator population. Applying Itô's formula to Eq. (3) leads to

$$
\begin{aligned}
& d \ln x=\left(a_{1}(t)-\frac{\alpha^{2}(t)}{2}-b_{1}(t) x-\frac{c_{1}(t) y}{m_{1}(t)+m_{2}(t) x+m_{3}(t) y}\right) d t+\alpha(t) d B_{1}(t) \\
& d \ln y=\left(-a_{2}(t)-\frac{\beta^{2}(t)}{2}-b_{2}(t) y+\frac{c_{2}(t) x}{m_{1}(t)+m_{2}(t) x+m_{3}(t) y}\right) d t+\beta(t) d B_{2}(t) .
\end{aligned}
$$

Let $r_{1}(t)=a_{1}(t)-\frac{\alpha^{2}(t)}{2}, r_{2}(t)=-a_{2}(t)-\frac{\beta^{2}(t)}{2}$, then $\left\langle r_{2}\right\rangle^{*}<0$. For the prey population $x(t)$ of system (3), we have

Theorem 3 (i) If $\left\langle r_{1}\right\rangle^{*}<0$, then the prey population $x(t)$ will go to extinction a.s.

(ii) If $\left\langle r_{1}\right\rangle^{*}=0$, then the prey population $x(t)$ will be non-persistent in the mean a.s.

(iii) If $\left\langle r_{1}\right\rangle^{*}>0$, then the prey population $x(t)$ will be weakly persistent in the mean a.s.

(iv) If $\left\langle r_{1}\right\rangle_{*}-\left\langle\frac{c_{1}(t)}{m_{3}(t)}\right\rangle^{*}>0$, then the prey population $x(t)$ will be strongly persistent in the mean a.s.

Proof (i) It follows from (10) that

$$
\begin{aligned}
\ln x(t)-\ln x_{0}= & \int_{0}^{t}\left[r_{1}(s)-b_{1}(s) x(s)-\frac{c_{1}(s) y(s)}{m_{1}(s)+m_{2}(s) x(s)+m_{3}(s) y(s)}\right] d s \\
& +\int_{0}^{t} \alpha(s) d B_{1}(s),
\end{aligned}
$$

$\ln x(t)-\ln x_{0} \leq \int_{0}^{t} r_{1}(s) d s+\int_{0}^{t} \alpha(s) d B_{1}(s)$, set $M_{1}(t)=\int_{0}^{t} \alpha(s) d B_{1}(s)$, it is a martingale whose quadratic variation is $\left\langle M_{1}, M_{1}\right\rangle_{t}=\int_{0}^{t} \alpha^{2}(s) d s \leq\left(\alpha^{u}\right)^{2} t$. Making use of the strong law of large numbers for martingale yields

$$
\lim _{t \rightarrow+\infty} \frac{M_{1}(t)}{t}=0, \quad \text { a.s. }
$$

then

$$
\frac{\ln x(t)-\ln x_{0}}{t} \leq \frac{1}{t} \int_{0}^{t} r_{1}(s) d s+\frac{M_{1}(t)}{t} .
$$

Taking superior limit on both sides of inequality (14) leads to $\lim \sup _{t \rightarrow+\infty} \frac{\ln x(t)}{t} \leq\left\langle r_{1}\right\rangle^{*}<0$, we can see that $\lim _{t \rightarrow+\infty} x(t)=0$.

(ii) By Eq. (12), we have

$$
\frac{\ln x(t)-\ln x_{0}}{t} \leq\left\langle r_{1}\right\rangle-b_{1}^{l}\langle x(t)\rangle+\frac{M_{1}(t)}{t} .
$$

It follows from the property of superior limit and (13) that for arbitrary $\varepsilon>0$, there exists $T>0$ such that $\left\langle r_{1}\right\rangle \leq\left\langle r_{1}\right\rangle^{*}+\frac{\varepsilon}{2}$ and $\frac{M_{1}(t)}{t} \leq \frac{\varepsilon}{2}$ for all $t \geq T$. Substituting these inequalities into (15) yields

$$
\ln x(t)-\ln x_{0} \leq\left(\left\langle r_{1}\right\rangle^{*}+\frac{\varepsilon}{2}\right) t-b_{1}^{l} \int_{0}^{t} x(s) d s+\frac{\varepsilon}{2} t \leq\left(\left\langle r_{1}\right\rangle^{*}+\varepsilon\right) t-b_{1}^{l} \int_{0}^{t} x(s) d s,
$$


when $\left\langle r_{1}\right\rangle^{*}=0$, then

$$
\ln \frac{x(t)}{x_{0}} \leq \varepsilon t-b_{1}^{l} x_{0} \int_{0}^{t} \frac{x(s)}{x_{0}} d s
$$

By $b_{1}^{l}>0$ and Lemma 3, we have $\langle x(t)\rangle^{*} \leq \frac{\varepsilon}{b_{1}^{l}}$, by virtue of the arbitrariness of $\varepsilon,\langle x(t)\rangle^{*} \leq 0$. Since the solution of system (3) is nonnegative, it is easy to have $\langle x(t)\rangle^{*}=0$, that is to say, the prey population $x(t)$ is non-persistent in the mean a.s.

(iii) We only need to show that there exists a constant $\mu_{1}>0$ such that for any solution $(x(t), y(t))$ of system (3) with initial value $\left(x_{0}, y_{0}\right) \in R_{+}^{2},\langle x(t)\rangle^{*} \geq \mu_{1}>0$ a.s. Otherwise, for arbitrary $\varepsilon_{1}>0$, there exists a solution $(\tilde{x}(t), \tilde{y}(t))$ with positive initial value $x_{0}>0$ and $y_{0}>0$ such that $P\left\{\langle\tilde{x}(t)\rangle^{*}<\varepsilon_{1}\right\}>0$.

Let $\varepsilon_{1}$ be sufficiently small so that

$$
\left\langle r_{1}\right\rangle^{*}-b_{1}^{u} \varepsilon_{1}>0, \quad\left\langle r_{2}\right\rangle^{*}+\frac{c_{2}^{u}}{m_{1}^{l}} \varepsilon_{1}<0 .
$$

It follows from Eq. (11) that

$$
\frac{\ln \tilde{y}(t)-\ln y_{0}}{t} \leq\left\langle r_{2}\right\rangle-b_{2}^{l}\langle\tilde{y}(t)\rangle+\frac{c_{2}^{u}}{m_{1}^{l}}\langle\tilde{x}(t)\rangle+\frac{M_{2}(t)}{t}
$$

here $M_{2}(t)=\int_{0}^{t} \beta(s) d B_{2}(s)$, it also has

$$
\lim _{t \rightarrow \infty} \frac{M_{2}(t)}{t}=0
$$

By virtue of (17), it leads to $\left[t^{-1} \ln \tilde{y}(t)\right]^{*} \leq\left\langle r_{2}\right\rangle^{*}+\frac{c_{2}^{u}}{m_{1}^{l}} \varepsilon_{1}<0$, thus

$$
\lim _{t \rightarrow \infty} \tilde{y}(t)=0 .
$$

On the other hand, it follows from Eq. (12) that

$$
\frac{\ln \tilde{x}(t)-\ln x_{0}}{t} \geq\left\langle r_{1}\right\rangle-b_{1}^{u}|\tilde{x}(t)\rangle-\frac{c_{1}^{u}}{m_{1}^{l}}\langle\tilde{y}(t)\rangle+\frac{M_{1}(t)}{t} .
$$

Taking the superior limit to the above inequality and making use of (13), (16) and (19), we have $\left[t^{-1} \ln \tilde{x}(t)\right]^{*} \geq\left\langle r_{1}\right\rangle^{*}-b_{1}^{u} \varepsilon_{1}>0$, that is to say, we have shown $P\left\{\left[t^{-1} \ln \tilde{x}(t)\right]^{*}>0\right\}>0$, this is a contradiction to Lemma 2. Therefore, $\langle x(t)\rangle^{*}>0$, the prey population $x(t)$ will be weakly persistent in the mean a.s.

(iv) By Eq. (10), we get

$$
d \ln x \geq\left(r_{1}(t)-b_{1}(t) x-\frac{c_{1}(t)}{m_{3}(t)}\right) d t+\alpha(t) d B_{1}(t) .
$$

It is easy to have

$$
\frac{\ln x(t)-\ln x_{0}}{t} \geq\left\langle r_{1}\right\rangle-\left\langle\frac{c_{1}(t)}{m_{3}(t)}\right\rangle-b_{1}^{u}|x(t)\rangle+\frac{M_{1}(t)}{t} .
$$


If $\left\langle r_{1}\right\rangle_{*}-\left\langle\frac{c_{1}(t)}{m_{3}(t)}\right\rangle^{*}>0$, there exists sufficiently small $\varepsilon>0$ such that $\left\langle r_{1}\right\rangle_{*}-\left\langle\frac{c_{1}(t)}{m_{3}(t)}\right\rangle^{*}-\varepsilon>0$. It follows from the property of superior limit, interior limit and (13) that for positive number $\varepsilon$, there exists a $T>0$ such that

$$
\left\langle r_{1}\right\rangle>\left\langle r_{1}\right\rangle_{*}-\frac{\varepsilon}{3}, \quad\left\langle\frac{c_{1}(t)}{m_{3}(t)}\right\rangle<\left\langle\frac{c_{1}(t)}{m_{3}(t)}\right\rangle^{*}+\frac{\varepsilon}{3}, \quad \frac{M_{1}(t)}{t}>-\frac{\varepsilon}{3}
$$

for all $t>T$. Then

$$
\begin{aligned}
\frac{\ln x(t)-\ln x_{0}}{t} & \geq\left\langle r_{1}\right\rangle_{*}-\frac{\varepsilon}{3}-\left\langle\frac{c_{1}(t)}{m_{3}(t)}\right\rangle^{*}-\frac{\varepsilon}{3}-b_{1}^{u}|x(t)\rangle-\frac{\varepsilon}{3} \\
& \geq\left(\left\langle r_{1}\right\rangle_{*}-\left\langle\frac{c_{1}(t)}{m_{3}(t)}\right\rangle^{*}-\varepsilon\right)-b_{1}^{u}|x(t)\rangle .
\end{aligned}
$$

By virtue of Lemma 3 and the arbitrariness of $\varepsilon$, we have

$$
\langle x(t)\rangle_{*} \geq \frac{\left\langle r_{1}\right\rangle_{*}-\left\langle\frac{c_{1}(t)}{m_{3}(t)}\right\rangle^{*}}{b_{1}^{u}}>0 .
$$

In other words, the prey population $x(t)$ is strongly persistent in the mean a.s.

Remark 1 The results of Theorem 3 illustrate that $\left\langle r_{1}\right\rangle^{*}$ is the threshold between weak persistence in the mean and extinction. Note $r_{1}(t)=a_{1}(t)-\frac{\alpha^{2}(t)}{2}$, if $\frac{\alpha^{2}(t)}{2}>a_{1}(t)$, then the prey population will be extinct, no matter whether there are predators. However, the prey population will survive when not considering environmental noise. This indicates that when the density of environmental noise is larger than the intrinsic growth rate of prey, it will cause the extinction of prey population. Therefore, it is more suitable to take into account stochastic perturbation in the systems. Here we can also find that the condition (iv) implies condition (iii), that is to say, the prey population must be weakly persistent in the mean when it is strongly persistent in the mean.

For the predator population, we have the following result.

Theorem 4 (i) If $\left(b_{1}\right)_{*}\left\langle r_{2}\right\rangle^{*}+\left(\frac{c_{2}(t)}{m_{1}(t)}\right)^{*}\left\langle r_{1}\right\rangle^{*}<0$, then the predator population $y(t)$ will go to extinction a.s.

(ii) If $\left(b_{1}\right)_{*}\left\langle r_{2}\right\rangle^{*}+\left(\frac{c_{2}(t)}{m_{1}(t)}\right)^{*}\left\langle r_{1}\right\rangle^{*}=0$, then the predator population $y(t)$ will be non-persistent in the mean a.s.

(iii) If $\left\langle r_{2}\right\rangle^{*}+\left\langle\frac{c_{2}(t) \bar{x}(t)}{m_{1}(t)+m_{2}(t) \bar{x}(t)+m_{3}(t) \bar{y}(t)}\right\rangle^{*}>0$, then the predator population $y(t)$ will be weakly persistent in the mean a.s. where $(\bar{x}(t), \bar{y}(t))$ is the solution of Eq. (8) with initial value $\left(x_{0}, y_{0}\right) \in R_{+}^{2}$.

(iv) If $\left\langle r_{2}\right\rangle^{*}+\left\langle\frac{c_{2}(t)}{m_{2}(t)}\right\rangle^{*}>0$, then the predator population $y(t)$ has a superior bound in time average, that is, $\langle y(t)\rangle^{*} \leq \frac{\left\langle r_{2}\right\rangle^{*}+\left\langle\frac{c_{2}(t)}{m_{2}(t)}\right\rangle^{*}}{b_{2}^{l}}$.

Proof (i) If $\left\langle r_{1}\right\rangle^{*} \leq 0$, then it follows from Theorem 3 that $\langle x(t)\rangle^{*}=0$. By Eq. (11),

$$
\frac{\ln y(t)-\ln y_{0}}{t} \leq\left\langle r_{2}\right\rangle+\frac{c_{2}^{u}}{m_{1}^{l}}\langle x(t)\rangle+\frac{M_{2}(t)}{t}
$$

therefore, $\left[t^{-1} \ln y(t)\right]^{*} \leq\left\langle r_{2}\right\rangle^{*}<0$, then $\lim _{t \rightarrow \infty} y(t)=0$. 
Now, if $\left\langle r_{1}\right\rangle^{*}>0$, it follows from the property of superior limit, interior limit and (13) that for sufficiently small $\varepsilon$, there exists a $T>0$ such that

$$
\begin{aligned}
\frac{\ln x(t)-\ln x_{0}}{t} & \leq\left\langle r_{1}\right\rangle-\left(b_{1}\right)_{*}\langle x(t)\rangle+\frac{M_{1}(t)}{t} \\
& \leq\left\langle r_{1}\right\rangle^{*}+\frac{\varepsilon}{2}-\left(b_{1}\right)_{*}|x(t)\rangle+\frac{\varepsilon}{2}
\end{aligned}
$$

for all $t>T$. Applying Lemma 3 and the arbitrariness of $\varepsilon$ yield

$$
\langle x(t)\rangle^{*} \leq \frac{\left\langle r_{1}\right\rangle^{*}}{\left(b_{1}\right)_{*}} .
$$

Substituting the above inequality into (11) gives

$$
\begin{aligned}
{\left[t^{-1} \ln y(t)\right]^{*} } & \leq\left\langle r_{2}\right\rangle^{*}+\left\langle\frac{c_{2}(t)}{m_{1}(t)} x(t)\right\rangle^{*} \\
& \leq\left\langle r_{2}\right\rangle^{*}+\left(\frac{c_{2}(t)}{m_{1}(t)}\right)^{*}\langle x(t)\rangle^{*} \\
& \leq\left\langle r_{2}\right\rangle^{*}+\left(\frac{c_{2}(t)}{m_{1}(t)}\right)^{*} \frac{\left\langle r_{1}\right\rangle^{*}}{\left(b_{1}\right)_{*}},
\end{aligned}
$$

then

$$
\left[t^{-1} \ln y(t)\right]^{*} \leq \frac{\left(b_{1}\right)_{*}\left\langle r_{2}\right\rangle^{*}+\left(\frac{c_{2}(t)}{m_{1}(t)}\right)^{*}\left\langle r_{1}\right\rangle^{*}}{\left(b_{1}\right)_{*}}<0
$$

which means $\lim _{t \rightarrow \infty} y(t)=0$ a.s.

(ii) In the case (i), we have shown that if $\left\langle r_{1}\right\rangle^{*} \leq 0$, then $\lim _{t \rightarrow \infty} y(t)=0$, therefore, $\langle y(t)\rangle^{*}=0$. Now, we will prove that $\langle y(t)\rangle^{*}=0$ is still valid when $\left\langle r_{1}\right\rangle^{*}>0$. Otherwise, if $\langle y(t)\rangle^{*}>0$, then it follows from Lemma 2 that $\left[t^{-1} \ln y(t)\right]^{*}=0$. Making use of (21), one can see that

$$
0=\left[t^{-1} \ln y(t)\right]^{*} \leq\left\langle r_{2}\right\rangle^{*}+\left(\frac{c_{2}(t)}{m_{1}(t)}\right)^{*}\langle x(t)\rangle^{*}
$$

On the other hand, for arbitrary $\varepsilon>0$, there exists a $\bar{T}>0$ such that

$$
\left\langle r_{2}\right\rangle<\left\langle r_{2}\right\rangle^{*}+\frac{\varepsilon}{3}, \quad\left\langle\frac{c_{2}(t)}{m_{1}(t)} x(t)\right\rangle<\left(\frac{c_{2}(t)}{m_{1}(t)}\right)^{*}\langle x(t)\rangle^{*}+\frac{\varepsilon}{3}, \quad \frac{M_{2}(t)}{t}<\frac{\varepsilon}{3}
$$

for all $t>\bar{T}$. Substituting these inequalities into (11) yields

$$
\begin{aligned}
\frac{\ln y(t)-\ln y_{0}}{t} & \leq\left\langle r_{2}\right\rangle+\left\langle\frac{c_{2}(t)}{m_{1}(t)} x(t)\right\rangle-\left\langle b_{2}(t) y(t)\right\rangle+\frac{M_{2}(t)}{t} \\
& \leq\left\langle r_{2}\right\rangle^{*}+\frac{\varepsilon}{3}+\left(\frac{c_{2}(t)}{m_{1}(t)}\right)^{*}\langle x(t)\rangle^{*}+\frac{\varepsilon}{3}-\left(b_{2}\right)_{*}\langle y(t)\rangle+\frac{\varepsilon}{3} \\
& \leq\left\langle r_{2}\right\rangle^{*}+\varepsilon+\left(\frac{c_{2}(t)}{m_{1}(t)}\right)^{*}\langle x(t)\rangle^{*}-\left(b_{2}\right)_{*}|y(t)\rangle .
\end{aligned}
$$


Then an application of Lemma 3 and (22) results in $\langle y(t)\rangle^{*} \leq \frac{\left\langle r_{2}\right\rangle^{*}+\varepsilon+\left(\frac{c_{2}(t)}{m_{1}(t)}\right)^{*}\langle x(t)\rangle^{*}}{\left(b_{2}\right)_{*}}$. By virtue of (20) and the arbitrariness of $\varepsilon$,

$$
\langle y(t)\rangle^{*} \leq \frac{\left\langle r_{2}\right\rangle^{*}\left(b_{1}\right)_{*}+\left(\frac{c_{2}(t)}{m_{1}(t)}\right)^{*}\left\langle r_{1}\right\rangle^{*}}{\left(b_{1}\right)_{*}\left(b_{2}\right)_{*}}=0,
$$

which is a contradiction to our assumption, therefore, $\langle y(t)\rangle^{*}=0$ a.s.

(iii) In the following, we need to show that $\langle y(t)\rangle^{*}>0$ a.s. Otherwise, for arbitrary $\varepsilon_{2}>0$, there exists a solution $(\hat{x}(t), \hat{y}(t))$ of system (3) with positive initial value $\left(x_{0}, y_{0}\right) \in R_{+}^{2}$ such that $P\left\{\langle\hat{y}(t)\rangle^{*}<\varepsilon_{2}\right\}>0$. Let $\varepsilon_{2}$ be sufficiently small so that

$$
\left\langle r_{2}\right\rangle^{*}+\left\langle\frac{c_{2}(t) \bar{x}(t)}{m_{1}(t)+m_{2}(t) \bar{x}(t)+m_{3}(t) \bar{y}(t)}\right\rangle^{*}>\left(b_{2}^{u}+\frac{2 c_{1}^{u} c_{2}^{u}}{b_{1}^{l}\left(m_{1}^{l}\right)^{2}}\right) \varepsilon_{2} .
$$

It follows from (11) that

$$
\begin{aligned}
\frac{\ln \hat{y}(t)-\ln y_{0}}{t}= & \left\langle r_{2}\right\rangle+\left\langle\frac{c_{2}(t) \bar{x}(t)}{m_{1}(t)+m_{2}(t) \bar{x}(t)+m_{3}(t) \bar{y}(t)}\right\rangle-\left\langle b_{2}(t) \hat{y}(t)\right\rangle+\frac{M_{2}(t)}{t} \\
& +\left\langle\frac{c_{2}(t) \hat{x}(t)}{m_{1}(t)+m_{2}(t) \hat{x}(t)+m_{3}(t) \hat{y}(t)}-\frac{c_{2}(t) \bar{x}(t)}{m_{1}(t)+m_{2}(t) \bar{x}(t)+m_{3}(t) \bar{y}(t)}\right\rangle .
\end{aligned}
$$

Here $(\bar{x}(t), \bar{y}(t))$ is the solution of model (8) with initial value $\left(x_{0}, y_{0}\right) \in R_{+}^{2}$ and $\hat{x}(t) \leq \bar{x}(t)$, $\hat{y}(t) \leq \bar{y}(t)$, a.s. for $t \in[0,+\infty)$.

Because of

$$
\begin{aligned}
\frac{c_{2}(t) \hat{x}(t)}{m_{1}(t)+m_{2}(t) \hat{x}(t)+m_{3}(t) \hat{y}(t)}-\frac{c_{2}(t) \bar{x}(t)}{m_{1}(t)+m_{2}(t) \bar{x}(t)+m_{3}(t) \bar{y}(t)} \\
=\frac{c_{2}(t) m_{3}(t) \bar{x}(\bar{y}-\hat{y})-c_{2}(t) m_{1}(t)(\bar{x}-\hat{x})-c_{2}(t) m_{3}(t) \bar{y}(\bar{x}-\hat{x})}{\left(m_{1}(t)+m_{2}(t) \hat{x}(t)+m_{3}(t) \hat{y}(t)\right)\left(m_{1}(t)+m_{2}(t) \bar{x}(t)+m_{3}(t) \bar{y}(t)\right)} \\
\geq \frac{c_{2}(t) m_{3}(t) \bar{x}(\bar{y}-\hat{y})}{\left(m_{1}(t)+m_{2}(t) \hat{x}(t)+m_{3}(t) \hat{y}(t)\right)\left(m_{1}(t)+m_{2}(t) \bar{x}(t)+m_{3}(t) \bar{y}(t)\right)} \\
\quad-\frac{c_{2}(t) m_{1}(t)}{m_{1}^{2}(t)}(\bar{x}-\hat{x})-\frac{c_{2}(t) m_{3}(t) \bar{y}}{m_{1}(t) m_{3}(t) \bar{y}}(\bar{x}-\hat{x}) \\
\geq-\frac{2 c_{2}(t)}{m_{1}(t)}(\bar{x}-\hat{x})
\end{aligned}
$$

then

$$
\begin{aligned}
\frac{\ln \hat{y}(t)-\ln y_{0}}{t} \geq & \left\langle r_{2}\right\rangle+\left\langle\frac{c_{2}(t) \bar{x}(t)}{m_{1}(t)+m_{2}(t) \bar{x}(t)+m_{3}(t) \bar{y}(t)}\right\rangle-\left\langle b_{2}(t) \hat{y}(t)\right\rangle+\frac{M_{2}(t)}{t} \\
& -\left\langle\frac{2 c_{2}(t)}{m_{1}(t)}(\bar{x}(t)-\hat{x}(t))\right\rangle \\
\geq & \left\langle r_{2}\right\rangle+\left\langle\frac{c_{2}(t) \bar{x}(t)}{m_{1}(t)+m_{2}(t) \bar{x}(t)+m_{3}(t) \bar{y}(t)}\right\rangle-\left\langle b_{2}(t) \hat{y}(t)\right\rangle+\frac{M_{2}(t)}{t} \\
& -\left\langle\frac{2 c_{2}^{u}}{m_{1}^{l}}(\bar{x}(t)-\hat{x}(t))\right\rangle .
\end{aligned}
$$


Consider the Lyapunov function $V_{2}(t)=|\ln \bar{x}(t)-\ln \hat{x}(t)|$, then $V_{2}(t)$ is a positive function on $R_{+}$, by Itô's formula, (8) and (10), we have

$$
\begin{aligned}
d^{+} V_{2}(t) & =\left[-b_{1}(t)(\bar{x}(t)-\hat{x}(t))+\frac{c_{1}(t) \hat{y}(t)}{m_{1}(t)+m_{2}(t) \hat{x}(t)+m_{3}(t) \hat{y}(t)}\right] d t \\
& \leq\left[\frac{c_{1}^{u}}{m_{1}^{l}} \hat{y}(t)-b_{1}^{l}(\bar{x}(t)-\hat{x}(t))\right] d t .
\end{aligned}
$$

Integrating from 0 to $t$ and dividing by $t$ on both sides of the inequality yield

$$
\frac{V_{2}(t)-V_{2}(0)}{t} \leq \frac{c_{1}^{u}}{m_{1}^{l}}\langle\hat{y}(t)\rangle-b_{1}^{l}\langle\bar{x}(t)-\hat{x}(t)\rangle
$$

Owing to $\frac{V_{2}(t)}{t} \geq 0$, it leads to

$$
b_{1}^{l}\langle\bar{x}(t)-\hat{x}(t)\rangle \leq \frac{c_{1}^{u}}{m_{1}^{l}}\langle\hat{y}(t)\rangle+\frac{V_{2}(0)}{t},
$$

here $V_{2}(0)=0$, then $\langle\bar{x}(t)-\hat{x}(t)\rangle \leq \frac{c_{1}^{u}}{m_{1}^{l} b_{1}^{l}}\langle\hat{y}(t)\rangle$. Substituting the above inequality into (24), we have

$$
\begin{aligned}
\frac{\ln \hat{y}(t)-\ln y_{0}}{t} \geq & \left\langle r_{2}\right\rangle+\left\langle\frac{c_{2}(t) \bar{x}(t)}{m_{1}(t)+m_{2}(t) \bar{x}(t)+m_{3}(t) \bar{y}(t)}\right\rangle \\
& -\left\langle b_{2}(t) \hat{y}(t)\right\rangle+\frac{M_{2}(t)}{t}-\frac{2 c_{1}^{u} c_{2}^{u}}{b_{1}^{l}\left(m_{1}^{l}\right)^{2}}\langle\hat{y}(t)\rangle .
\end{aligned}
$$

Taking superior limit of the above inequality, we get

$$
\left[t^{-1} \ln \hat{y}(t)\right]^{*} \geq\left\langle r_{2}\right\rangle^{*}+\left\langle\frac{c_{2}(t) \bar{x}(t)}{m_{1}(t)+m_{2}(t) \bar{x}(t)+m_{3}(t) \bar{y}(t)}\right\rangle^{*}-\left(b_{2}^{u}+\frac{2 c_{1}^{u} c_{2}^{u}}{b_{1}^{l}\left(m_{1}^{l}\right)^{2}}\right) \varepsilon_{2}>0,
$$

which contradicts Lemma 2, then $\langle y(t)\rangle^{*}>0$ a.s., that is to say, the predator population $y(t)$ is weakly persistent in the mean a.s.

(iv) It follows from Eq. (11) that

$$
d \ln y \leq\left(r_{2}(t)-b_{2}(t) y+\frac{c_{2}(t)}{m_{2}(t)}\right) d t+\beta(t) d B_{2}(t)
$$

that is to say,

$$
\frac{\ln y(t)-\ln y_{0}}{t} \leq\left\langle r_{2}\right\rangle+\left\langle\frac{c_{2}(t)}{m_{2}(t)}\right\rangle-b_{2}^{l}\langle y(t)\rangle+\frac{M_{2}(t)}{t} .
$$

The following proof is similar to the proof of (iv) in Theorem 3, here we omit it.

Remark 2 From the proof of Theorem 4, we can observe that if $\left\langle r_{1}\right\rangle^{*}<0$, then $\left(b_{1}\right)_{*}\left\langle r_{2}\right\rangle^{*}+$ $\left(\frac{c_{2}(t)}{m_{1}(t)}\right)^{*}\left\langle r_{1}\right\rangle^{*}<0$ is straightforward. It shows that if the prey population goes to extinction, the predator population will also go to extinction, which is consistent with the reality. In the other case, $\left\langle r_{1}\right\rangle^{*}>0,\left(b_{1}\right)_{*}\left\langle r_{2}\right\rangle^{*}+\left(\frac{c_{2}(t)}{m_{1}(t)}\right)^{*}\left\langle r_{1}\right\rangle^{*}<0$, which means the prey population 
will survive, but the predator population will go to extinction. Notice $r_{2}(t)=-a_{2}(t)-\frac{\beta^{2}(t)}{2}$, this phenomenon may be caused by the large death rate of predator $a_{2}(t)$ or the noise density $\beta^{2}(t)$.

\section{Global attractiveness of the solution}

In this section, we give sufficient conditions of global attractiveness.

Definition 3 System (3) is said to be globally attractive if

$$
\lim _{t \rightarrow \infty}\left|x_{1}(t)-x_{2}(t)\right|=\lim _{t \rightarrow \infty}\left|y_{1}(t)-y_{2}(t)\right|=0 \quad \text { a.s. }
$$

where $\left(x_{1}(t), y_{1}(t)\right)$ and $\left(x_{2}(t), y_{2}(t)\right)$ are two arbitrary positive solutions of system (3) with initial values $\left(x_{10}, y_{10}\right) \in R_{+}^{2}$ and $\left(x_{20}, y_{20}\right) \in R_{+}^{2}$.

To give the result of global attractiveness, we show some lemmas first.

Lemma 4 [26] Suppose that an $n$-dimensional stochastic process $X(t)$ on $t \geq 0$ satisfies the condition

$$
E|X(t)-X(s)|^{\alpha_{1}} \leq c|t-s|^{1+\alpha_{2}}, \quad 0 \leq s, t<\infty
$$

for some positive constants $\alpha_{1}, \alpha_{2}$ and c. Then there exists a continuous modification $\tilde{X}(t)$ of $X(t)$ which has the property that for every $\vartheta \in\left(0, \alpha_{2} / \alpha_{1}\right)$, there is a positive random variable $h(\omega)$ such that

$$
P\left\{\omega: \sup _{0<|t-s|<h(\omega), 0 \leq s, t<\infty} \frac{|\tilde{X}(t, \omega)-X(t, \omega)|}{|t-s|^{\vartheta}} \leq \frac{2}{1-2^{-\vartheta}}\right\}=1 .
$$

In other words, almost every sample path of $\tilde{X}(t)$ is locally but uniformly Hölder continuous with an exponent $\vartheta$.

Lemma 5 Let $(x(t), y(t))$ be a solution of system (3) on $t \geq 0$ with initial value $\left(x_{0}, y_{0}\right) \in R_{+}^{2}$, then almost every sample path of $(x(t), y(t))$ is uniformly continuous.

Proof Equation (3) is equivalent to the following stochastic integral equation:

$$
\begin{aligned}
x(t)= & x_{0}+\int_{0}^{t} x(s)\left[a_{1}(s)-b_{1}(s) x(s)-\frac{c_{1}(s) y(s)}{m_{1}(s)+m_{2}(s) x(s)+m_{3}(s) y(s)}\right] d s \\
& +\int_{0}^{t} \alpha(s) x(s) d B_{1}(s) .
\end{aligned}
$$

Let $f(s)=x(s)\left[a_{1}(s)-b_{1}(s) x(s)-\frac{c_{1}(s) y(s)}{m_{1}(s)+m_{2}(s) x(s)+m_{3}(s) y(s)}\right], g(s)=\alpha(s) x(s)$, notice that

$$
\begin{aligned}
E|f(t)|^{p} & =E\left|x\left(a_{1}(t)-b_{1}(t) x-\frac{c_{1}(t) y}{m_{1}(t)+m_{2}(t) x+m_{3}(t) y}\right)\right|^{p} \\
& =E\left[|x|^{p}\left|a_{1}(t)-b_{1}(t) x-\frac{c_{1}(t) y}{m_{1}(t)+m_{2}(t) x+m_{3}(t) y}\right|^{p}\right]
\end{aligned}
$$




$$
\begin{aligned}
& \leq \frac{1}{2} E|x|^{2 p}+\frac{1}{2} E\left|a_{1}(t)-b_{1}(t) x-\frac{c_{1}(t) y}{m_{1}(t)+m_{2}(t) x+m_{3}(t) y}\right|^{2 p} \\
& \leq \frac{1}{2} E|x|^{2 p}+\frac{1}{2} E\left|a_{1}^{u}+b_{1}^{u} x+\frac{c_{1}^{u}}{m_{1}^{l}} y\right|^{2 p} \\
& \leq \frac{1}{2} E|x|^{2 p}+\frac{1}{2} 3^{2 p-1}\left[\left(a_{1}^{u}\right)^{2 p}+\left(b_{1}^{u}\right)^{2 p} E|x|^{2 p}+\left(\frac{c_{1}^{u}}{m_{1}^{l}}\right)^{2 p} E|y|^{2 p}\right] \\
& \leq \frac{1}{2} K_{1}(2 p)+\frac{3^{2 p-1}}{2}\left[\left(a_{1}^{u}\right)^{2 p}+\left(b_{1}^{u}\right)^{2 p} K_{1}(2 p)+\left(\frac{c_{1}^{u}}{m_{1}^{l}}\right)^{2 p} K_{2}(2 p)\right] \\
& =: F(p), \\
E|g(t)|^{p} & =E|\alpha(t) x(t)|^{p} \leq\left(\alpha^{u}\right)^{p} E|x|^{p} \leq\left(\alpha^{u}\right)^{p} K_{1}(p)=: G(p) .
\end{aligned}
$$

Moreover, in view of the moment inequality for stochastic integrals in [24, p.39], one can obtain that for $0 \leq t_{1} \leq t_{2}$ and $p>2$,

$$
\begin{aligned}
E\left|\int_{t_{1}}^{t_{2}} g(s) d B_{1}(s)\right|^{p} & \leq\left[\frac{p(p-1)}{2}\right]^{\frac{p}{2}}\left(t_{2}-t_{1}\right)^{\frac{(p-2)}{2}} \int_{t_{1}}^{t_{2}} E|g(s)|^{p} d s \\
& \leq\left[\frac{p(p-1)}{2}\right]^{\frac{p}{2}}\left(t_{2}-t_{1}\right)^{\frac{p}{2}} G(p) .
\end{aligned}
$$

Then for $0<t_{1}<t_{2}<\infty, t_{2}-t_{1} \leq 1, \frac{1}{p}+\frac{1}{q}=1$, we have

$$
\begin{array}{rl}
E & x\left(t_{2}\right)-\left.x\left(t_{1}\right)\right|^{p} \\
& =E\left|\int_{t_{1}}^{t_{2}} f(s) d s+\int_{t_{1}}^{t_{2}} g(s) d B_{1}(s)\right|^{p} \\
& \leq 2^{p-1} E\left|\int_{t_{1}}^{t_{2}} f(s) d s\right|^{p}+2^{p-1} E\left|\int_{t_{1}}^{t_{2}} g(s) d B_{1}(s)\right|^{p} \\
& \leq 2^{p-1}\left(\int_{t_{1}}^{t_{2}} 1^{q} d s\right)^{\frac{p}{q}} E\left(\int_{t_{1}}^{t_{2}}|f(s)|^{p} d s\right)+2^{p-1}\left[\frac{p(p-1)}{2}\right]^{\frac{p}{2}}\left(t_{2}-t_{1}\right)^{\frac{p}{2}} G(p) \\
& \leq 2^{p-1}\left(t_{2}-t_{1}\right)^{\frac{p}{q}} F(p)\left(t_{2}-t_{1}\right)+2^{p-1}\left[\frac{p(p-1)}{2}\right]^{\frac{p}{2}}\left(t_{2}-t_{1}\right)^{\frac{p}{2}} G(p) \\
& \leq 2^{p-1}\left(t_{2}-t_{1}\right)^{p} F(p)+2^{p-1}\left[\frac{p(p-1)}{2}\right]^{\frac{p}{2}}\left(t_{2}-t_{1}\right)^{\frac{p}{2}} G(p) \\
& \leq 2^{p-1}\left(t_{2}-t_{1}\right)^{\frac{p}{2}}\left\{\left(t_{2}-t_{1}\right)^{\frac{p}{2}}+\left[\frac{p(p-1)}{2}\right]^{\frac{p}{2}}\right\} M(p) \\
& \leq 2^{p-1}\left(t_{2}-t_{1}\right)^{\frac{p}{2}}\left\{1+\left[\frac{p(p-1)}{2}\right]^{\frac{p}{2}}\right\} M(p),
\end{array}
$$

where $M(p)=F(p)+G(p)$, it follows from Lemma 4 that almost every sample path of $x(t)$ is locally but uniformly Hölder continuous with an exponent $\vartheta$ for $\vartheta \in\left(0, \frac{p-2}{2 p}\right)$ and therefore almost every sample path of $x(t)$ is uniformly continuous on $t \in R_{+}$. In the same way, we can demonstrate that almost every sample path of $y(t)$ is uniformly continuous. 
Lemma 6 [27] Let $f$ be a nonnegative function defined on $R_{+}$such that $f$ is integrable and is uniformly continuous, then $\lim _{t \rightarrow \infty} f(t)=0$.

Theorem 5 System (3) is globally attractive if

$$
b_{1}^{l}-\frac{2 c_{2}^{u}}{m_{1}^{l}}-\frac{c_{1}^{u} m_{2}^{u}}{m_{1}^{l} m_{3}^{l}}>0, \quad b_{2}^{l}-\frac{2 c_{1}^{u}}{m_{1}^{l}}>0
$$

Proof For $\left(x_{1}(t), y_{1}(t)\right)$ and $\left(x_{2}(t), y_{2}(t)\right)$, any two positive solutions of system (3) with initial values $\left(x_{10}, y_{10}\right) \in R_{+}^{2}$ and $\left(x_{20}, y_{20}\right) \in R_{+}^{2}$ define

$$
V(t)=\left|\ln x_{1}(t)-\ln x_{2}(t)\right|+\left|\ln y_{1}(t)-\ln y_{2}(t)\right|,
$$

then $V(t)$ is a continuous positive function on $t \geq 0$. A direct calculation of the right differential $d^{+} V(t)$ of $V(t)$, by (10) and (11) and Itô's formula,

$$
\begin{aligned}
& d^{+} V(t)=\operatorname{sgn}\left(x_{1}-x_{2}\right)\left\{\left[\frac{d x_{1}}{x_{1}}-\frac{\left(d x_{1}\right)^{2}}{2 x_{1}^{2}}\right]-\left[\frac{d x_{2}}{x_{2}}-\frac{\left(d x_{2}\right)^{2}}{2 x_{2}^{2}}\right]\right\} \\
& +\operatorname{sgn}\left(y_{1}-y_{2}\right)\left\{\left[\frac{d y_{1}}{y_{1}}-\frac{\left(d y_{1}\right)^{2}}{2 y_{1}^{2}}\right]-\left[\frac{d y_{2}}{y_{2}}-\frac{\left(d y_{2}\right)^{2}}{2 y_{2}^{2}}\right]\right\} \\
& =\left\{-b_{1}(t) \operatorname{sgn}\left(x_{1}-x_{2}\right)\left(x_{1}-x_{2}\right)-b_{2}(t) \operatorname{sgn}\left(y_{1}-y_{2}\right)\left(y_{1}-y_{2}\right)\right. \\
& -\operatorname{sgn}\left(x_{1}-x_{2}\right) \frac{c_{1}(t) m_{1}(t)\left(y_{1}-y_{2}\right)+c_{1}(t) m_{2}(t)\left[x_{2}\left(y_{1}-y_{2}\right)-y_{2}\left(x_{1}-x_{2}\right)\right]}{\left(m_{1}(t)+m_{2}(t) x_{1}+m_{3}(t) y_{1}\right)\left(m_{1}(t)+m_{2}(t) x_{2}+m_{3}(t) y_{2}\right)} \\
& \left.+\operatorname{sgn}\left(y_{1}-y_{2}\right) \frac{c_{2}(t) m_{1}(t)\left(x_{1}-x_{2}\right)-c_{2}(t) m_{3}(t)\left[x_{2}\left(y_{1}-y_{2}\right)-y_{2}\left(x_{1}-x_{2}\right)\right]}{\left(m_{1}(t)+m_{2}(t) x_{1}+m_{3}(t) y_{1}\right)\left(m_{1}(t)+m_{2}(t) x_{2}+m_{3}(t) y_{2}\right)}\right\} d t \\
& \leq\left\{-b_{1}(t)\left|x_{1}-x_{2}\right|-b_{2}(t)\left|y_{1}-y_{2}\right|\right. \\
& +\frac{c_{1}(t) m_{1}(t)\left|y_{1}-y_{2}\right|+c_{1}(t) m_{2}(t) x_{2}\left|y_{1}-y_{2}\right|+c_{1}(t) m_{2}(t) y_{2}\left|x_{1}-x_{2}\right|}{\left(m_{1}(t)+m_{2}(t) x_{1}+m_{3}(t) y_{1}\right)\left(m_{1}(t)+m_{2}(t) x_{2}+m_{3}(t) y_{2}\right)} \\
& \left.+\frac{c_{2}(t) m_{1}(t)\left|x_{1}-x_{2}\right|-c_{2}(t) m_{3}(t) x_{2}\left|y_{1}-y_{2}\right|+c_{2}(t) m_{3}(t) y_{2}\left|x_{1}-x_{2}\right|}{\left(m_{1}(t)+m_{2}(t) x_{1}+m_{3}(t) y_{1}\right)\left(m_{1}(t)+m_{2}(t) x_{2}+m_{3}(t) y_{2}\right)}\right\} d t \\
& \leq\left\{-b_{1}(t)\left|x_{1}-x_{2}\right|-b_{2}(t)\left|y_{1}-y_{2}\right|+\frac{c_{1}(t)}{m_{1}(t)}\left|y_{1}-y_{2}\right|+\frac{c_{1}(t)}{m_{1}(t)}\left|y_{1}-y_{2}\right|\right. \\
& \left.+\frac{c_{1}(t) m_{2}(t)}{m_{1}(t) m_{3}(t)}\left|x_{1}-x_{2}\right|+\frac{c_{2}(t)}{m_{1}(t)}\left|x_{1}-x_{2}\right|+\frac{c_{2}(t)}{m_{1}(t)}\left|x_{1}-x_{2}\right|\right\} d t \\
& \leq\left\{-\left(b_{1}(t)-\frac{2 c_{2}(t)}{m_{1}(t)}-\frac{c_{1}(t) m_{2}(t)}{m_{1}(t) m_{3}(t)}\right)\left|x_{1}-x_{2}\right|-\left(b_{2}(t)-\frac{2 c_{1}(t)}{m_{1}(t)}\right)\left|y_{1}-y_{2}\right|\right\} d t \\
& \leq\left\{-\left(b_{1}^{l}-\frac{2 c_{2}^{u}}{m_{1}^{l}}-\frac{c_{1}^{u} m_{2}^{u}}{m_{1}^{l} m_{3}^{l}}\right)\left|x_{1}-x_{2}\right|-\left(b_{2}^{l}-\frac{2 c_{1}^{u}}{m_{1}^{l}}\right)\left|y_{1}-y_{2}\right|\right\} d t \text {. }
\end{aligned}
$$

Integrating both sides leads to

$$
V(t) \leq V(0)-\int_{0}^{t}\left(b_{1}^{l}-\frac{2 c_{2}^{u}}{m_{1}^{l}}-\frac{c_{1}^{u} m_{2}^{u}}{m_{1}^{l} m_{3}^{l}}\right)\left|x_{1}(s)-x_{2}(s)\right|+\left(b_{2}^{l}-\frac{2 c_{1}^{u}}{m_{1}^{l}}\right)\left|y_{1}(s)-y_{2}(s)\right| d s .
$$


Consequently,

$$
V(t)+\int_{0}^{t}\left(b_{1}^{l}-\frac{2 c_{2}^{u}}{m_{1}^{l}}-\frac{c_{1}^{u} m_{2}^{u}}{m_{1}^{l} m_{3}^{l}}\right)\left|x_{1}(s)-x_{2}(s)\right|+\left(b_{2}^{l}-\frac{2 c_{1}^{u}}{m_{1}^{l}}\right)\left|y_{1}(s)-y_{2}(s)\right| d s \leq V(0)<\infty .
$$

It then follows from $V(t) \geq 0$ and (25) that

$$
\left|x_{1}(t)-x_{2}(t)\right| \in L^{1}[0,+\infty), \quad\left|y_{1}(t)-y_{2}(t)\right| \in L^{1}[0,+\infty) .
$$

Then from Lemmas 5 and 6, we get the desired assertion.

\section{Numerical simulation}

In this section, to support the main results in our paper, some simulation figures are introduced. Here we use the Milstein method mentioned in Higham [28] to simulate stochastic equations, considering the following discrete equations:

$$
\begin{aligned}
x_{k+1}= & x_{k}+x_{k}\left[a_{1}(k \Delta t)-b_{1}(k \Delta t) x_{k}-\frac{c_{1}(k \Delta t) y_{k}}{m_{1}(k \Delta t)+m_{2}(k \Delta t) x_{k}+m_{3}(k \Delta t) y_{k}}\right] \Delta t \\
& +\alpha(k \Delta t) x_{k} \sqrt{\Delta t} \xi_{k}+\frac{\alpha^{2}(k \Delta t)}{2} x_{k}\left(\xi_{k}^{2}-1\right) \Delta t, \\
y_{k+1}= & y_{k}+y_{k}\left[-a_{2}(k \Delta t)-b_{2}(k \Delta t) y_{k}+\frac{c_{2}(k \Delta t) x_{k}}{m_{1}(k \Delta t)+m_{2}(k \Delta t) x_{k}+m_{3}(k \Delta t) y_{k}}\right] \Delta t \\
& +\beta(k \Delta t) y_{k} \sqrt{\Delta t} \eta_{k}+\frac{\beta^{2}(k \Delta t)}{2} y_{k}\left(\eta_{k}^{2}-1\right) \Delta t,
\end{aligned}
$$

$\xi_{k}$ and $\eta_{k}(k=1,2, \ldots, n)$ are the Gaussian random variables $N(0,1)$. Let $a_{1}(t)=0.4+$ $0.01 \sin t, b_{1}(t)=0.7+0.01 \sin t, c_{1}(t)=0.3+0.02 \sin t, c_{2}(t)=0.1+0.02 \sin t, a_{2}(t)=$ $0.2+0.05 \sin t, b_{2}(t)=0.2+0.01 \sin t, m_{1}(t)=0.2+0.04 \sin t, m_{2}(t)=0.5+0.05 \sin t$, $m_{3}(t)=0.3+0.05 \sin t$ with initial value $\left(x_{0}, y_{0}\right)=(0.5,0.6)$. In Figure 1 , we choose $\frac{\alpha^{2}(t)}{2}=$ $0.5+0.02 \sin t, \frac{\beta^{2}(t)}{2}=0.3+0.02 \sin t$, then it is easy to have $\left\langle r_{1}\right\rangle^{*}=-0.1<0$. In view of Theorems 3 and 4, both prey population $x$ and predator population $y$ go to extinction. Figure 1 confirms this. In Figure 2, we choose $\frac{\alpha^{2}(t)}{2}=0.2+0.02 \sin t, \frac{\beta^{2}(t)}{2}=0.4+0.02 \sin t$, the other parameters are the same. At this time, we get $\left\langle r_{1}\right\rangle^{*}=0.2>0$ and $\left(b_{1}\right)_{*}\left\langle r_{2}\right\rangle^{*}+\left(\frac{c_{2}(t)}{m_{1}(t)}\right)^{*}\left\langle r_{1}\right\rangle^{*}=$ $-0.314<0$. By virtue of Theorems 3 and 4 , the prey population $x$ is weakly persistent in the mean and the predator population $y$ goes to extinction, which is shown in Figure 2.

In Figure 3, we choose $c_{1}(t)=0.1+0.02 \sin t, c_{2}(t)=0.2+0.02 \sin t, m_{1}=0.1+0.04 \sin t$, $\frac{\alpha^{2}(t)}{2}=0.02+0.02 \sin t, \frac{\beta^{2}(t)}{2}=0.02+0.02 \sin t$, the other parameters are the same; then $\left\langle r_{1}\right\rangle^{*}=0.38>0$, but $\left\langle r_{2}\right\rangle^{*}+\left\langle\frac{c_{2}(t) \bar{x}(t)}{m_{1}(t)+m_{2}(t) \bar{x}(t)+m_{3}(t) \bar{y}(t)}\right\rangle^{*}>0$ is difficult to verify at present. Figure 3 illustrates that the situation that both prey population $x$ and predator population $y$ are weakly persistent in the mean exists.

\section{Conclusions}

Owing to theoretical and practical importance, the predator-prey system with Beddington-DeAngelis functional response has received great attention and has been studied extensively, but for stochastic non-autonomous case, there is none. Here, we consider a stochastic non-autonomous predator-prey system with Beddington-DeAngelis functional 


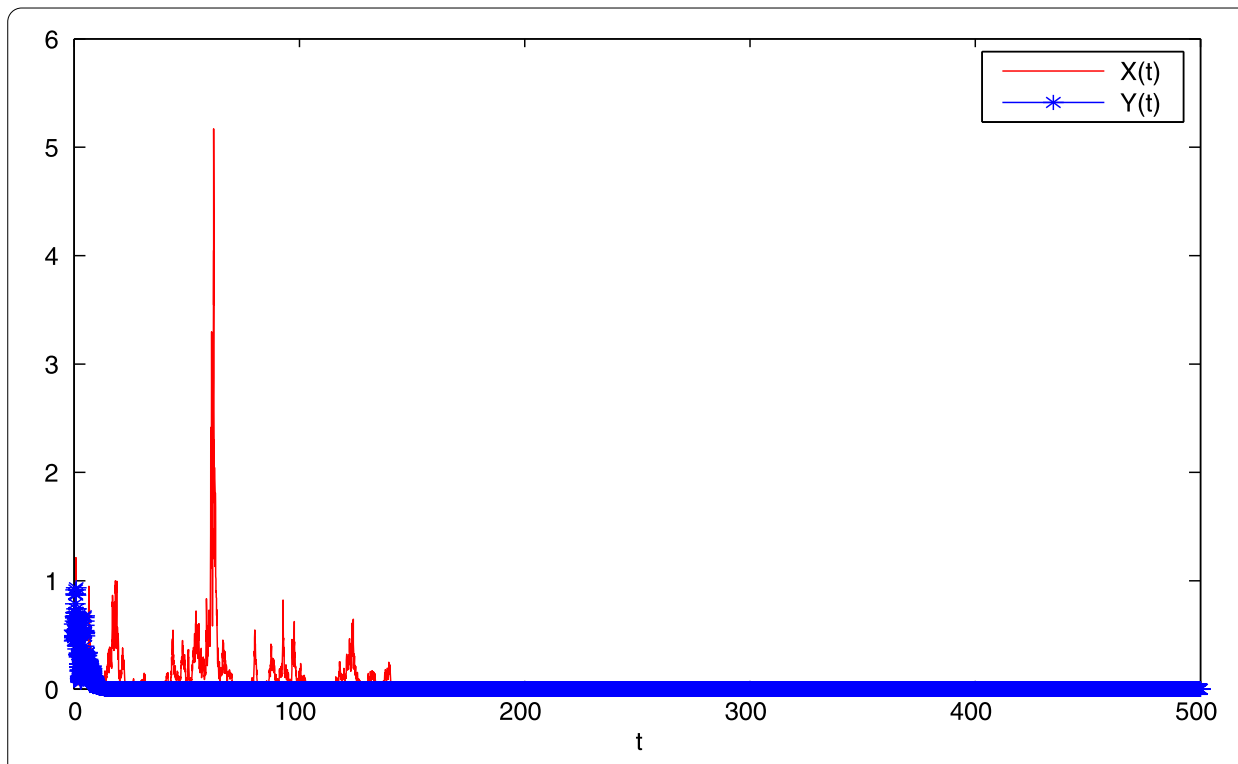

Figure 1 Both populations go to extinction. Solutions of system (3) for $\alpha^{2}(t)=1+0.04 \sin t$,

$\beta^{2}(t)=0.6+0.04 \sin t$, with step size $\Delta t=0.05>0$. Prey and predator populations are represented by red line and blue line with a star, respectively.

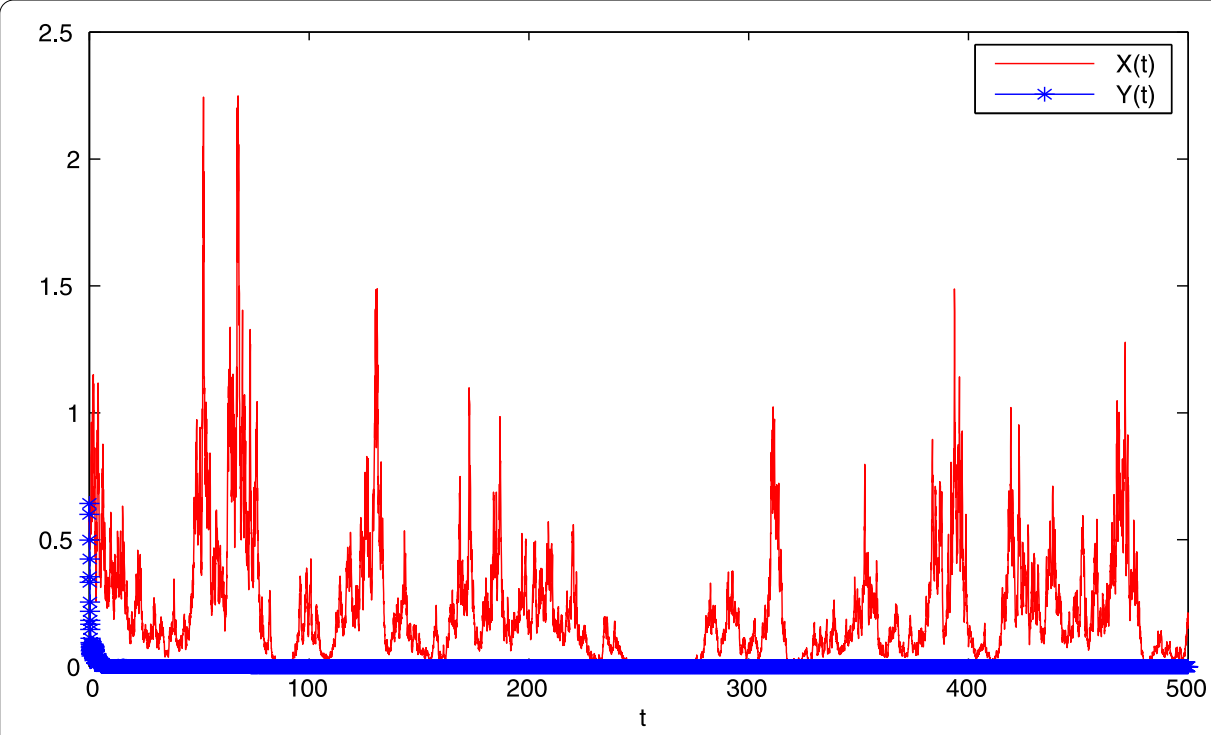

Figure 2 Prey is weakly persistent in the mean, predator goes to extinction. Solutions of system (3) for $\alpha^{2}(t)=0.4+0.04 \sin t, \beta^{2}(t)=0.8+0.04 \sin t$, with step size $\Delta t=0.05>0$. Prey and predator populations are represented by red line and blue line with a star, respectively.

response. Firstly, we show that the solution of system (3) is globally positive and stochastically ultimately bounded. Sufficient conditions for extinction, non-persistence in the mean, weak persistence in the mean and strong persistence in the mean are obtained. The threshold between weak persistence and extinction for prey population is established. We also show that the solution of system (3) is globally attractive under some sufficient conditions. These results are useful to estimate the risk of extinction of species in the system. Besides, global attractiveness means that all the species in the community can coexist. 


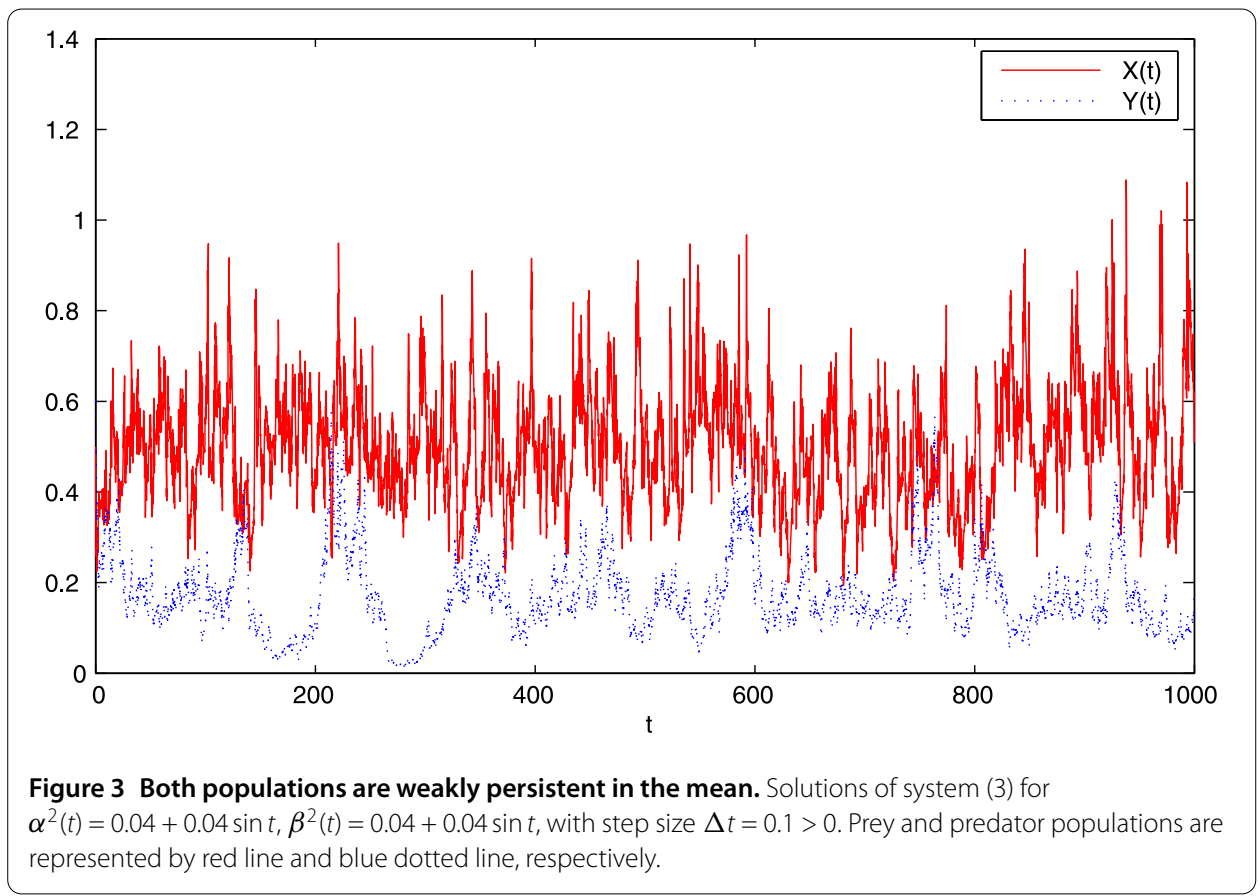

There are still some interesting questions deserved to be study. For example, here the condition of the weak persistence in the mean for predator is only a sufficient condition, which is not so ideal. Maybe we can give the threshold between weak persistence in the mean and extinction for predator in the future. Moreover, we can consider colored noise in the models owing to sudden environmental changes caused by seasons or other reasons.

\section{Competing interests}

The authors declare that they have no competing interests.

\section{Authors' contributions}

SL proposed the model and completed the main part of the manuscript. XZ checked all the results and polished the language. All authors read and approved the final manuscript.

\section{Acknowledgements}

This work was supported by National Science Foundation of China (Nos. 11071275 and 11228104) and by the Special Fund for Basic Scientific Research of Central Colleges (CCNU10B01005).

Received: 3 August 2012 Accepted: 3 January 2013 Published: 22 January 2013

\section{References}

1. Beddington, JR: Mutual interference between parasites or predators and its effect on searching efficiency. J. Anim. Ecol. 44, 331-340 (1975)

2. DeAngelis, DL, Goldstein, RA, O'Neill, RV: A model for trophic interaction. Ecology 56, 881-892 (1975)

3. Cantrell, RS, Consner, C: On the dynamics of predator-prey models with the Beddington-DeAngelis functional response. J. Math. Anal. Appl. 257, 206-222 (2001)

4. Hwang, TW: Global analysis of the predator-prey system with Beddington-DeAngelis functional response. J. Math. Anal. Appl. 281, 395-401 (2003)

5. Zeng, ZJ, Fan, M: Study on a non-autonomous predator-prey system with Beddington-DeAngelis functional response. Math. Comput. Model. 48, 1755-1764 (2008)

6. Fan, M, Kuang, Y: Dynamics of a nonautonomous predator-prey system with the Beddington-DeAngelis functional response. J. Math. Anal. Appl. 295, 15-39 (2004)

7. Li, HY, Takeuchi, Y: Dynamics of the density dependent predator-prey system with Beddington-DeAngelis functional response. J. Math. Anal. Appl. 374, 644-654 (2011)

8. Bahar, A, Mao, XR: Stochastic delay Lotka-Volterra model. J. Math. Anal. Appl. 292, 364-380 (2004)

9. Bahar, A, Mao, XR: Stochastic delay population dynamics. Int. J. Pure Appl. Math. 11, 377-400 (2004)

10. Gard, TC: Persistence in stochastic food web models. Bull. Math. Biol. 46, 357-370 (1984)

11. Gard, TC: Stability for multispecies population models in random environments. Nonlinear Anal. 10, 1411-1419 (1986) 
12. Mao, XR, Marion, G, Renshaw, E: Environmental Brownian noise suppresses explosions in population dynamics. Stoch. Process. Appl. 97, 95-110 (2002)

13. Ji, CY, Jiang, DQ, Shi, NZ: Analysis of a predator-prey model with modified Leslie-Gower and Holling-type II schemes with stochastic perturbation. J. Math. Anal. Appl. 359, 482-498 (2009)

14. Ji, CY, Jiang, DQ: Dynamics of a stochastic density dependent predator-prey system with Beddington-DeAngelis functional response. J. Math. Anal. Appl. 381, 441-453 (2011)

15. Liu, M, Wang, K: Global stability of a nonlinear stochastic predator-prey system with Beddington-DeAngelis functional response. Commun. Nonlinear Sci. Numer. Simul. 16, 1114-1121 (2011)

16. Ji, CY, Jiang, DQ, Li, XY: Qualitative analysis of a stochastic ratio-dependent predator-prey system. J. Comput. Appl. Math. 235, 1326-1341 (2011)

17. Rudnicki, R: Long-time behavior of a stochastic prey-predator model. Stoch. Process. Appl. 108, 93-107 (2003)

18. Jiang, DQ, Shi, NZ: A note on nonautonomous logistic equation with random perturbation. J. Math. Anal. Appl. 303, 164-172 (2005)

19. Jiang, DQ, Shi, NZ, Li, XY: Global stability and stochastic permanence of a non-autonomous logistic equation with random perturbation. J. Math. Anal. Appl. 340, 588-597 (2008)

20. Li, XY: Population dynamical behavior of non-autonomous Lotka-Volterra competitive system with random perturbation. Discrete Contin. Dyn. Syst. 24, 523-545 (2009)

21. Liu, $M$, Wang, K: Extinction and permanence in a stochastic non-autonomous population system. Appl. Math. Lett. 23, 1464-1467 (2010)

22. Liu, M, Wang, K: Persistence and extinction in stochastic non-autonomous logistic systems. J. Math. Anal. Appl. 375, 443-457 (2011)

23. Liu, M, Wang, K: Persistence, extinction and global asymptotical stability of a non-autonomous predator-prey model with random perturbation. Appl. Math. Model. 36, 5344-5353 (2012)

24. Mao, XY: Stochastic Differential Equations and Applications. Horwood, Chichester (1997)

25. Liu, M, Wang, K, Wu, Q: Survival analysis of stochastic competitive models in a polluted environment and stochastic competitive exclusion principle. Bull. Math. Biol. 73, 1969-2012 (2011)

26. Karatzas, I, Shreve, SE: Brownian Motion and Stochastic Calculus. Springer, Berlin (1991)

27. Barbălat, I: Systèmes d'équations différentielles d'oscillations non linéaires. Rev. Roum. Math. Pures Appl. 4, 267-270 (1959)

28. Higham, DJ: An algorithmic introduction to numerical simulation of stochastic differential equations. SIAM Rev. 43, 525-546 (2001)

doi:10.1186/1687-1847-2013-19

Cite this article as: Li and Zhang: Dynamics of a stochastic non-autonomous predator-prey system with

Beddington-DeAngelis functional response. Advances in Difference Equations 2013 2013:19.

\section{Submit your manuscript to a SpringerOpen ${ }^{\circ}$ journal and benefit from:}

- Convenient online submission

Rigorous peer review

- Immediate publication on acceptance

- Open access: articles freely available online

- High visibility within the field

- Retaining the copyright to your article 